\title{
Colección de tesis de la Universidad del Cusco sustentadas en el rectorado del Dr. Albert A. Giesecke (1910-1923), ubicadas en la Biblioteca del Instituto Riva Agüero
}

\author{
Mercedes Giesecke Sara Lafosse \\ mgieseckes@unmsm.edu.pe \\ Universidad Nacional Mayor de San Marcos \\ Facultad de Ciencias Sociales
}

\section{RESUMEN:}

Esta es una breve presentación de carácter bibliográfico archivístico, de un listado de ochenta tesis históricas que tiene la Colección Giesecke en la biblioteca del Instituto Riva Agüero. Salvo dos de ellas, todas las demás son de la Universidad del Cusco, escritas durante la época del rectorado de Giesecke que se desarrolló entre 1910 y 1923, la mayor parte fue para obtener el grado de doctor y algunas para el bachillerato. Cuya clasificación temática incluye aspectos sobre el matrimonio, la educación, el problema indígena, estudios geológicos, geográficos y económicos, estudios sobre el desarrollo regional del Cusco y sobre el derecho. Palabras Clave: Tesis Universidad del Cusco; 1911 a 1922; Colección Alberto Giesecke; Reforma universitaria.

\section{University of Cusco collection of theses sustained in the rectorship of Dr. Albert A. Giesecke (1910-1923) found in the Institute of Riva Aguero Library}

\begin{abstract}
:
This is a brief presentation of a review on filed bibliography, about eighty historic theses registered in the shelves of Giesecke's Book Collection in the Riva Agüero's Institute Library. Except for dissertations, the others are from the University of Cusco, sustained in the rectorship of Dr. Giesecke between 1910 and 1923, to fulfill mainly the requirements for the degree of Doctor, including some Bachelor theses. Research topics include marriage, education, indigenous problems, geology, geographic and economic studies; also Cusco's regional development and law.

Keywords: University of Cusco theses; 1911 to 1922; Alberto Giesecke's book collection; University reform.
\end{abstract}


Esta es una breve presentación de un conjunto de tesis universitarias encontradas en la Colección del doctor Albert Giesecke y que considero de interés general para todo aquel interesado en conocer más sobre el contexto histórico y pedagógico de la universidad del Cusco y la región, entre 1910 y 1923. Previamente se hará una breve descripción de la vida y formación profesional de Giesecke lo que fue valorado para ser seleccionado dentro del equipo de educadores que fue contratado por el Perú y contribuyó con los logros académicos alcanzados en su gestión como rector en la Universidad del Cusco. Para esto, se ha realizado una concisa reseña biográfica del Dr. Albert Giesecke consultando su historia oral hecha por la Universidad de Columbia, así como algunos otros documentos guardados en su colección.

Giesecke nació el 30 de noviembre de 1883 en Philadelphia, allí estudió la educación elemental en el Paschal Grammar School, para luego ingresar al Central High School en Philadelphia. Aquí estudio en el recientemente fundado Departamento de Comercio, inaugurado por el mismo Theodore Roosevelt. (Mason, 1963, p. 16)

Dentro de las especialidades que ofrecía este Departamento, Giesecke optó por estudiar taquigrafía técnica, lo que le permitió desde muy joven ayudar todos los sábados a su profesor, el Dr. Cheeseman A. Herrick en su investigación sobre los ferrocarriles en América del Sur; quien a su vez apoyaba a Giesecke en las materias escolares y le daba instrucción sobre historia, geografía y economía de América del Sur. Se graduó con honores del Central High School en 1902 a los 19 años de edad. Eran dos en competencia entre 141 graduados y solo podía haber un ganador, como fue el segundo en aprovechamiento, a manera de compensación recibió una beca para estudiar en Pennsylvania University. (Mason, 1963, p. 17) Tuvo dos años de español, tres años de alemán y dos años de latín en el Central High School.

Al ingresar a la universidad, le convalidaron algunos cursos básicos, técnicos y de comercio que había llevado en el Central High School, los que fueron aceptados como trabajos introductorios en el Wharton School de la Universidad de Pennsylvania. (Mason, 1963, p. 20) Eso le permitió terminar la carrera en menos de 4 años. Entre los cursos y catedráticos que tuvo el Dr. Giesecke se encuentran los que figuran en el Cuadro 1.

En esta universidad estudió Ciencias Políticas con Leo S. Rowe, quien luego se convirtió en el director de la Unión Panamericana. Según Giesecke, Rowe era una persona amable, agradable y entendible en sus ponencias en el Wharton School,su perfil profesional le sirvió para ser considerado entre los más destacados expertos sobre Latinoamérica, por lo que era llamado para asesorar sobre temas legales y administrativos en asuntos internacionales con países latinoamericanos (Mason, 1963, p. 21). Durante su etapa universitaria Giesecke fue un estudiante destacado y se ganó la confianza de varios de sus maestros, entre ellos, la del Dr. Rowe, quien le encomendó su curso de Derecho Internacional y Privado de Latinoamérica para que lo enseñara a fines de 1908 y en el primer semestre de 1909 en la Universidad de Pennsylvania, y fue Rowe quien le recomendó para que trabaje en el Ministerio de Instrucción Pública del Perú (Mason, 1963, p. 22). Tal como testimonió Giesecke:

Yo fui recomendado para la administración y la reforma universitaria. Yo trabajé en la formación comercial del Central High School, lo que me calificaba para trabajar en la modificación de la secundaria comercial en el Perú. Así fui contratado por Perú para trabajar en el Perú por dos años (Mason, 1963, p. 22). 
Cuadro 1.

Educación Superior de Giesecke en la Universidad de Pennsylvania (Mason, 1963)

\begin{tabular}{|c|c|}
\hline Cursos & Profesor \\
\hline Economía Política & $\begin{array}{l}\text { Simón Patten, director de la Sección de Economía. Quien propuso la teoría del proteccionismo } \\
\text { como base en la actividad económica, quien se doctoró en Alemania y estuvo imbuido de la } \\
\text { significación económica, política y social que se veía ahí; influyó en Albert A. Giesecke (Mason, } \\
1963, \text { p. 22) }\end{array}$ \\
\hline Ciencia Política & Leo S. Rowe, quien luego dirigió la Unión Panamericana (Mason, 1963, p. 20) \\
\hline $\begin{array}{l}\text { Transporte; Traslado de Mercancías; } \\
\text { Estadística (Mason, 1963, p. 33) }\end{array}$ & Emory Johnson, decano del Wharton School (Mason, 1963, p. 23) \\
\hline Estadística & Walter Wilcox (Mason, 1963, p. 23) \\
\hline Economía; Comercio & Meade (Mason, 1963, p. 25) \\
\hline Juicios; Luchas laborales & Young (Mason, 1963, p. 26) \\
\hline Geografía & Paul Goode (Mason, 1963, p. 26) \\
\hline Historia Americana & $\begin{array}{l}\text { John O. Mac Master, Herman Ames, Learned y Wesselshaft (Mason, 1963, p. 26) } \\
\text { Charles Hall (Mason, 1963, p. 33) }\end{array}$ \\
\hline
\end{tabular}

La preparación universitaria de Giesecke se realizó con dicho plan de estudios, un caso ejemplar de la mejor enseñanza comercial liberal otorgada en una de las cuatro instituciones de enseñanza superior más prestigiosas de los Estados Unidos de Norteamérica para su época (Ver: James, 1910). Es importante destacar su aprendizaje en economía política, ciencia política, estadística, leyes y luchas laborales, e historia americana, tanto en la educación secundaria comercial como en la superior, cursos que le sirvieron de base para ser seleccionado como especialista en educación comercial y ser contratado por el Perú.

En la Universidad de Pennsylvania obtuvo el bachiller en Ciencias Económicas en 1904, a continuación, hizo los estudios de postgrado, logrando obtener el doctorado con la tesis en el estudio de Las sesiones de las Legislaturas de la Colonia en la Sociedad de Historia Colonial, así como en el Bureau of Statistics entre 1904 y 1905. (Giesecke, ¿1951?)

Según los datos por él consignados en su manuscrito (Giesecke, ¿1951?), estudió en Europa un semestre en el postgrado de la Universidad de Berlín, llevando seminarios como el de Economía, conducido por el profesor Gustav Schmoller, jefe de la Escuela Histórica de Economía Política, el de Finanzas, conducido por el profesor Adolf Wagner, y el de comercio con Max Sering. De manera complementaria participó dentro de las tareas del Servicio Social bajo los auspicios de la Administración de la Caridad del Concejo Municipal de Berlín. De Berlín pasó a París ingresando a La Sorbonne bajo la dirección de Leroy Beauliau. De París se trasladó a Lausanne en Suiza, en donde tuvo de maestro a Paretto, allí también hizo estudios en Economía, Finanzas y en Ciencia Política. Finalmente, entre 1906 y 1908 estudió su doctorado en la Universidad de Cornell, su investigación para la tesis doctoral lo llevó a investigar en los archivos del British Museum en Londres.

A la par que estudiaba, enseñaba economía y ciencia política en Cornell; luego redactó y defendió su tesis, lo que le permitió recibir el grado de $\mathrm{PhD}$ en Letras en la Universidad de Cornell; con una tesis sobre «Las Leyes Comerciales de Estados Unidos antes de 1789"o «American Commercial Legislation before 1789», que fue sustentada en 1908 y publicada en 1910. Toda esta sólida experiencia académica y desarrollo profesional obtenido en los EE.UU., le servirá como marco de referencia para aplicarla y aportar en la enseñanza comercial y la reforma educativa en el Perú. 
Cuando Albert Giesecke llega al Perú, el país se encontraba gobernado por el Partido Civilista, que tuvo en Augusto Bernardino Leguía la persona que concretaría su proyecto político. Leguía no era un desconocido en la gestión gubernamental, antes había sido dos veces ministro de hacienda, tanto en la presidencia de Manuel de la Puente Candamo (1903) como en la de José Pardo y Barreda, ambos del Partido Civilista. Su habilidad para los negocios locales, exportaciones de materias primas y sus lazos comerciales establecidos con los capitales norteamericanos durante su etapa como empresario, le sirvió para consolidar la dirección de la economía peruana con este país. Asimismo, por haber tenido éxito en este rubro, Leguía consideró que una de las mejores vías para el desarrollo del país debía estar ligada a una educación que permita el libre desenvolvimiento de las personas en base a un pensamiento liberal. Para ello era importante instruirse en la cuestión comercial y sus herramientas. Ya desde su primer mandato daba luces de lo que sería su discurso durante los once años en el poder: la construcción de una Patria Nueva.

El contexto del pensamiento liberal de Leguía, pudo estar asociado a la tendencia dominante del pensamiento de la época. El filósofo James Kloppenberg (1986) quien hizo un estudio de las ideas sobre la social democracia y progresivismo en el pensamiento europeo y norteamericano, decía que hubo una cierta coincidencia en las preocupaciones de los intelectuales,

...la comunalidad de sus pensamientos (de ciertos pensadores) forjó una "comunidad intelectual"entre pensadores quienes, sin conocerse entre sí, hicieron preguntas filosóficas y políticas muy similares entre 1870 y 1920, se hallaron a sí mismos como "vecinos dentro de un territorio de nuevas ideas". El liberalismo clásico fue transformado por cada uno de estos pensadores, quienes, también defendieron las libertades individuales y muy continuamente, la propiedad privada y cuestionaron los excesos del capitalismo industrial (Kloppenberg, 1986, p. 101).

El ambiente intelectual de esa época exigía una renovación pedagógica, tanto en sus postulados teóricos como en su metodología de enseñanza, es así que corrientes como el positivismo francés y el utilitarismo de origen anglosajón, dialogaban y tenían puntos de encuentro con el progresivismopedagógico norteamericano.

El progresivismo era también una postura pedagógica, la cual era abierta a toda propuesta del conocimiento que contribuyera al bien común. Englobaba toda ciencia basada en el rigor; en este sentido y como se sabe, por un lado, el positivismo se manifiesta a favor de una sociedad organizada en base a los preceptos y principios que emanan de la ciencia, como único instrumento para validar las acciones humanas en búsqueda del progreso y su método científico, pero también debería ser considerado en la resolución de dilemas morales, políticos y religiosos. En la sociedad industrial tecnificada, las personas deberían ser instruidas para tener una cualificación acorde a la exigencia de la sociedad, que le permitiese a su vez desenvolverse y desarrollarse.

Mientras el positivismo creyó encontrar en la ciencia la llave para el desarrollo sostenido de la sociedad en su conjunto, el utilitarismo como propuesta filosófica y pedagógica, de la mano de Jeremy Bentham(1748-1832), sostiene que el fin último de toda organización social es brindar las condiciones necesarias para la máxima satisfacción de sus necesidades. Una pedagogía es útil y buena si contribuye a la consecución del bien común, es decir, de toda la comunidad donde se desarrolla. Lo que se conoce como útil sería análogo a valioso y equivalente a la felicidad. Es un modelo paradigmático para todos los planos de la vida, basados en la maximización de hacer cosas buenas, útiles, cercanas a los intereses de la población. Según Nicola Abbagnano y Visalberghi, A. (1964:369) Bentham asume el utilitarismo como: 
(...) principio fundamental que el fin de toda actividad y de toda organización social consiste en la mayor felicidad posible del mayor número posible de personas. En virtud de esta máxima, una acciónes buena cuando es útil, es decir, cuando contribuye en mayor o menor medida a la felicidad común, procurando un placer y evitando un dolor (1964:369)

Estas dos corrientes de pensamiento eran contemporáneas a la pedagogía progresiva de la cual se nutrió Albert Giesecke. La pedagogía progresiva, fundamentada en la filosofía pragmatista de John Dewey, se hace patente en la escuela activa, que tiene como núcleo teórico poner al estudiante en el centro de todo el proceso de enseñanza - aprendizaje, donde la escuela es comprendida como un entorno o espacio de preparación para la vida. La escuela activa estimula una enseñanza basada en el desarrollo pleno del individuo, no como un ser aislado, sino, como pieza matriz de la organización social.

Si el hombre debe ser preparado para la vida y este corresponde a la naturaleza, entonces, ambos deben ser estudiados. La escuela nueva en sentido también es experimental, es sujeto de experimentación cotidiana, creándose una didáctica en el hacer, ideas que van a ser aplicadas por el Dr. Albert Giesecke en su trabajo como rector y docente en el Cusco, insistiendo a los estudiantes investigar sobre la realidad social de la región.

Es así que en 1909 el Ministro de Justicia e Instrucción, Manuel Vicente Villarán, a pedido del presidente Leguía, delegó a Francisco García Calderón la búsqueda de pedagogos calificados para ser contratados dentro del Plan de Modernización de la Educación Peruana. Esto se efectuó en mayo de 1909, cuando A. Giesecke recibió un telegrama del Dr. Francisco García Calderón para prestar servicios educativos en el Perú bajo la dirección del Ministerio de Instrucción, por un término de dos años prorrogable por el acuerdo de las partes.

García Calderón era embajador de Perú en Francia, y a su paso por los EE. UU. le pidió a Leo S. Rowe, que le ayudase en la selección de un especialista que se encargaría de la reforma de la educación comercial en el Perú. García Calderón fue a la Universidad de Pennsylvania porque allí se impartía una de las primeras cátedras sobre educación comercial a través de la escuela Wharton. Conversó con el profesor Rowe, amigo de Manuel Vicente Villarán, quien le recomendó a su asistente y ex alumno de dicha casa de estudios, con apenas 25 años, Albert Giesecke, para que asuma esa responsabilidad con un extraordinario equipo de docentes recientemente egresados de la Universidad de Columbia, quienes lo acompañaron en esta misión (Giesecke, 2015, p.372).

Según Giesecke, en EEUU hasta 1902 no hubo mucho interés por Latinoamérica ni por el castellano. Hasta que de pronto y liderado por especialistas, se hicieron informes sobre Latinoamérica en conferencias especializadas.

Ya en el Perú, la Comisión de Instrucción Pública de 1910 distribuyó las tareas para cada uno de los cuatro miembros de la misión educativa norteamericana: el Dr. Giesecke, en marzo de 1910, fue designado como rector para la Universidad del Cusco, en paralelo, fue asesor externo de la comisión especial de instrucción pública creada en 1910. Esto luego de haber introducido con éxito, una reforma de la educación comercial en el Colegio Nuestra Señora de Guadalupe.

El señor Harry Erwin Bard fue el secretario de la comisión especial de instrucción pública, quien investigó sobre la educación superior. El profesor Joseph Lockey se dedicó a la dirección de Inspección de la Instrucción Pública Primaria en Lima y Callao. El cuarto miembro de dicha misión, el profesor Joseph Mc Knight, llegó a ser el director de la Escuela Normal de Varones de Lima, quien viajó a Puno como Inspector Departamental de Instrucción Pública, época que 
compartió con José Antonio Encinas para reformar la Escuela 881, cuando este último fue director de dicha escuela primaria.

En marzo de 1910, con 26 años de edad, llegó al Cusco nombrado rector por el propio presidente de la República, encargándole de manera personal reabrir la Universidad Nacional de San Antonio Abad que permanecía cerrada por el gobierno debido a las demandas de los estudiantes. Estos se habían levantado contra los anticuados métodos de enseñanza, la corrupción, el compadrazgo y los malos manejos por parte de los docentes y autoridades administrativas de dicha casa de estudio:

El nombramiento de Albert A. Giesecke desató enconadas críticas políticas, hasta la medida que tuvo que sortear el pedido de interpelación del Ministro de Educación, por parte del Congreso de la República. Al parecer la ley universitaria de entonces exigía tener 30 años para ser rector. Finalmente, el jefe de gabinete renunció, pero por razones distintas a esa designación, y el asunto espinoso del gringo nombrado Rector en Cusco pasó a segundo plano (Valer, 2013, p. 1).

Desde el primer día que Giesecke pisó la ciudad, fue recibido con recelo, por parte las familias y autoridades conservadoras cusqueñas y algunos estudiantes, al considerarlo ajeno a las costumbres e idiosincrasia propias de esta parte del país. Un incidente que representa este clima social fue promovido por los ex profesores de la universidad del Cusco, quienes habían perdido sus puestos ante las exigencias de mejoras académicas por parte de la población estudiantil. Para obstaculizar por segundo año consecutivo, la reapertura de la universidad y el inicio de clases en 1910, los docentes llegaron a pedir expresamente:

....al prefecto de Cusco, y también al ministro de guerra en Lima, que dediquen el edificio en donde funciona la universidad para que sirva como cuartel, para ser utilizado por los voluntarios que vienen de las 13 provinciasalrededor de Cusco, porque es el único lugar disponible (Mason, 1963, p. 120).

Albert Giesecke estaba enterado quiénes eran los verdaderos responsables de este hecho gracias a la información brindada por un estudiante que trabajaba en la prefectura, quien no quería perder un año más de estudio. Por ello, dado que los docentes fueron expulsados de la universidad como consecuencia de la huelga estudiantil, se mantenían como enemigos de la Universidad y de los estudiantes. El panorama se complicaba aún más debido al respaldo de las principales familias del Cusco a la medida adoptada, quienes eran antiguos profesores o familiares de estos (Mason, 1963, p. 120).

Ante tal situación, Giesecke remitió un telegrama al presidente Leguía contándole las principales razones detrás de estos incidentes y solicitándole volver a tener el control de la casa de estudios, quien inmediatamente atendió su pedido. La efectividad a su solicitud se debió a que previo a su llegada al Cusco pidió como condición tener un trato directo con el presidente. Esta rápida acción por medio de telegramas, le brindó legitimidad ante las autoridades locales, estudiantes y población en general como gestor eficiente (Valer, 2013, p.2).

A pesar de la oposición de las familias conservadoras de la ciudad, Albert Giesecke pensó que era adecuado aceptar a cualquier persona dentro del proceso de admisión a la Universidad, esto sin importar su condición socio económica, antecedente familiar, oficio o género, siempre y cuando demostrase su aptitud y capacidad para estudiar, pagar la tasa correspondiente al derecho de admisión y obtener una vacante (Mason, 1963, p. 121). 
Bajo su gestión también se promovió que las mujeres puedan asistir a la universidad en el mayor número posible. Él logró que la Universidad admitiera a todas las mujeres que postulasen e ingresasen para recibir una educación superior, y así obtengan sus títulos y grados académicos, a pesar de haber una gran oposición por parte de las mujeres conservadoras de la ciudad, quienes sostenían que el lugar de las mujeres estaba en la casa y no en los espacios públicos (Mason, 1963, pp. 137 - 139).

La característica del nuevo rector no solo se reducía al trabajo académico, sinoque también dio un gran impulso a los deportes y apoyó las actividades extracurriculares de los estudiantes. La cercanía con los ellos se reflejaba al compartir algunos partidos de fútbol, tenis y básquet desde las primeras horas del día. «Con suscripciones voluntarias de catedráticos i alumnos se ha logrado arreglar una parte del patio del local para destinarla a algunos ejercicios de Sport como Lawn Tennis, Basket-Ball, Hand Ball, etc....» (Aparicio Vega, 2012, p.141 Centenario de la generación La Sierra. Fondo editorial de la ANR. Lima - Perú. citado en Giesecke, M., 2015.)

También colaboró activamente en la organización del Primer Congreso Nacional de Estudiantes del Perú, que se realizó en el Cusco en 1920. El mismo Víctor Raúl Haya de la Torre, presidente del cuerpo estudiantil de la Universidad San Marcos, le pidió que intercediera ante el presidente Leguía para que les diera el permiso respectivo. Consiguió que el gobierno les dotara de pasajes y viáticos para los estudiantes, además Giesecke facilitó los alojamientos de estudiantes en la ciudad durante el tiempo que durase el congreso:

Esa tarde, inmediatamente envié una cable y telegrama a las autoridades de la Universidad en el Cusco, y también para el Prefecto, y les pedí que asegurasen el alojamiento para 100 personas que iban a Cusco, para el Primer Congreso Nacional de Estudiantes, el primero que se llevara a cabo por los estudiantes universitarios en Perú.(...) Alojé tres de los estudiantes en mi casa, porque quería mantener un control sobre el cuerpo estudiantil, ya que pensé que esto podría ser deseable. Esos tres estudiantes fueron, en primer lugar, Victor Haya de la Torre, presidente del congreso; Dr. Jorge Basadre ... era el segundo de los estudiantes en el comité designado para venir a mi casa. El tercero era el hijo de un ex miembro del Gabinete y miembro de la Corte Suprema. El primero de ellos fue, por supuesto, el estudiante clave, ya que fue él quien tuvo la idea de hacer que me llame el presidente y conseguir que autorice el inicio del Congreso (Mason, 1963, p.146).

Por su formación académica, promovió los estudios de la realidad social de la región del Cusco a partir del trabajo de campo, buscando información económica de primera mano para el desarrollo de reflexiones orientadas a relacionar los desafíos con el desarrollo social. La información recogida era debatida dentro de las clases a la luz de los enfoques teóricos liberales modernizantes, lo que contribuyó en la redacción de monografías innovadoras y comprometidas con una política de recomendaciones.

La metodología activa de enseñanza utilizada por Giesecke como catedrático en jurisprudencia, economía y estadística, estaba de acuerdo a la corriente pedagógica de Dewey y que sus colegas emularon posteriormente, era manejada como una estrategia para lograr un aprendizaje dinámico, participativo y arraigado a los problemas del entorno regional. Los informes de las investigaciones de campo fueron publicados de forma resumida en la Revista Universitaria que empezó a circular en 1912. Más tarde el Dr. Giesecke les sugirió a muchos de sus alumnos que estos informes monográficos podrían ser usados como insumos para la obtención del grado de bachiller, Giesecke señaló: «fuimos reconocidos como una universidad líder en el Perú, a causa de esas actividades y estudios. He trabajado de esa manera. Y las alumnas, por supuesto, no son ya discriminadas, lo quisieran o no». (Mason, 1963, p.166) 
Para él cualquier ocasión era motivo de aprendizaje, como fue el caso del primer censo del siglo XX en el Cusco, donde los estudiantes aprendieron el uso de las técnicas estadísticas en base al curso de estadística que él dictaba. Durante el censo estuvo a cargo de verificar todo el proceso a caballo por las comunidades: «tuvimos más de 200 estudiantes que trabajaron sobre el área del censo y antes de lo cual todos trabajamos en casos de una muestra para obtener una idea previa de cómo iba ser» (Mason, 1963, p.162).

En cuanto a lo académico introdujo nuevos métodos y técnicas de enseñanza, dio orientación moderna a las cátedras que estuvieron a su cargo, tales como Psicología Experimental, Economía Política y Finanzas, dictadas para las Facultades de Ciencias Económicas y Jurisprudencia. (...) Indujo al alumnado a "escribir sobre lo que uno viera y comprobara"... de acuerdo al pragmatismo de W. James, muy en boga por entonces, cuyo principio original es el de determinar el significado de la verdad por su utilidad práctica. Esta orientación pragmática revolucionó el clima espiritual de la época y condujo a tomar interés en el estudio del pasado incaico y el presente indígena exaltando las glorias y valores nacionales que antes se tenían en el olvido, estudiando, a decir de Valcárcel: "todo lo relacionado con el Cusco". Inclusive reglamentó las tesis universitarias para que traten sobre temas originales y propios de nuestra realidad regional. (Gutiérrez citado en Giesecke, 2015, p. 167)

Basadre escribió lo siguiente: Pero ¿cuál era la situación desde la perspectiva de los alumnos ya conmovidos por la reforma Universitaria, la Revolución mexicana y la Revolución rusa? Los estudiantes cusqueños, entre los cuales se sentían ya las inquietudes creadas por las nuevas ideas tendrían su apoteosis en el primer Congreso de la Federación de Estudiantes del Perú celebrado en el Cusco del 11 al 20 de marzo de 1920, (... en el cual) «los estudiantes de San Marcos acostumbrados a la lejanía aristocrática del rector (Javier Prado) y a la distancia insalvable entre profesores y alumnos, quedarían asombrados de la democracia fraterna, informal y abierta que reinaba en San Antonio Abad». (...) Percibieron asimismo los frutos de la política de Giesecke, los problemas nacionales, sobre todo el del indio, eran ya el tema dominante de la universidad cusqueña en 1920. Como dice Basadre: «fue el descubrimiento brusco del mundo indígena que entonces era ignorado o despreciado en Lima». (Basadre citado por Giesecke, M., 2015 La Historia y la vidade Jorge Basadre, páginas 150 y siguientes. 1968. Historia de la República del Perú. 1822 - 1933. Tomo XIII. Ed. Universitaria.)

El prestigio, modernidad y avance democrático de la universidad cusqueña de la década del 20 cuenta con un testimonio insospechable a su favor. El de José Carlos Mariátegui. En los «Siete Ensayos», Mariátegui dice: «Si el movimiento renovador se muestra precariamente detenido en las Universidades de Lima, prospera en cambio en la Universidad del Cusco, donde la élite del profesorado acepta y sanciona los principios sustentados por los alumnos. Testimonio de esto es el anteproyecto de reorganización de la Universidad del Cusco, formulado por la Comisión que con este encargo formó el Gobierno (...) » (Citado pot Gutiérrez, 2010).

Tamayo dice: Hoy se hace difícil comprender cómo un puñado de profesores $(20$ - 30) y 165 alumnos (1922) pudieron realizar una tarea vasta, casi sobre humana. La única explicación posible está en su entrega apasionada y desinteresada a la cultura, en su amor por la ciencia y por el Cusco. Los impulsaba la llama de una vocación y no el simple afán por un salario. Ahí está el secreto de esa gran generación; su gran capacidad de hacer está en la enorme dimensión de su fe (Tamayo 1978, pp. 132-136, citado por Giesecke, 2015, pp. 601 y 602).

Otro tema que apasionaba al doctor Giesecke fue las manifestaciones culturales andinas, por lo que impulsó el estudio de los monumentos y restos arqueológicos para la puesta en valor 
del pasado histórico de la ciudad y su gente; en ese sentido, proporcionó los cimientos de los intelectuales indigenistas o de la llamada generación de la sierra, lo cual se potenció con el descubrimiento de Machu Picchu en 1911. Esto le valió pertenecer a prestigiosas instituciones académicas como miembro de la American Association of Anthropology desde 1912 y como presidente fundador de la Sociedad Geográfica del Cusco.

A su trabajo como rector, docente y promotor cultural en la Universidad del Cusco, le siguió la de examinador y jurado de tesis. Él estaba presente en casi todas las sustentaciones, tanto para el grado de bachiller como sobre todo para el grado de doctor. Giesecke era el responsable de dar el Visto Bueno a todas las tesis presentadas, para ello se dio expresamente mediante su reglamentación, en la que se precisaba que los temas a investigar muestren la realidad socioeconómica, cultural, geográfica, educativa y propuestas de desarrollo de la región. Esta labor le permitió obtener una copia de cada trabajo sustentado, gran parte de las tesis se encuentran en su colección.

Durante su permanencia en el Cusco también fue concejal en tres periodos a lo largo de doce años, colaboró en darle a esa ciudad Imperial más brillo y renombre, siendo Cusco uno de los lugares de orgullo del Perú. En esta ciudad se casó con doña Esther Matto Usandivaras quien fuera prima hermana de Clorinda Grimanesa Matto Usandivaras de Turner. Al culminar su último periodo como rector, en 1923, regresó a Lima.

Luego de haber concluido su gestión en el Cusco, asumió el cargo de Director General de Enseñanza entre 1924 y 1930. Interrumpido por un año de licencia cuando participó en la Comisión plebiscitaria en el año 1925. En relación a su nombramiento como Director General de Enseñanza, el Doctor Plácido Jiménez, ex ministro de educación del Perú en 1915 (MINEDU, 2017), le escribió en una misiva (Imagen 1) lo siguiente:

He visto con agrado su nombramiento de Director General de Enseñanza (...) conoce usted el sur del Perú, está usted penetrado de lo que es nuestra sierra y de la verdadera condición del indígena, sabe cómo son nuestros políticos y tiene probado su afecto al Perú. Con aquellos elementos y con su reconocida ilustración y laboriosidad, puede usted hacer mucho efectivo por el progreso de la instrucción (...) (Jiménez, 1923, s/n).

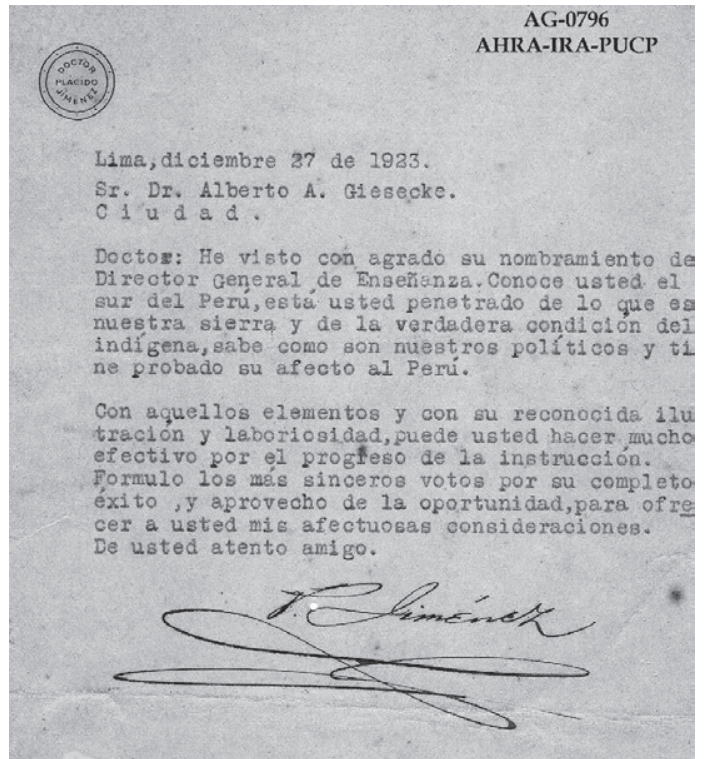

Imagen 1. Saludo de P. Jiménez al Dr. Albert Giesecke por su nombramiento como Director General de Enseñanza. 
Al término de su cargo en la Dirección General de Enseñanza en Perú, fue funcionario en la Embajada de los EE.UU. de Norteamérica por 35 años, honrado con varias distinciones por los esfuerzos desarrollados en pro de la cooperación oficial entre ambos países, desarrollando lazos oficiales y amistades personales en el campo de la educación, la ciencia y la cultura.

El Dr. Albert Giesecke falleció en el año 1968, en la ciudad de Lima, dejando una gran cantidad de bibliografía, cartas, diplomas y algunos artículos de valor que fue atesorando. Varios años después, la Dra. Margarita Giesecke, su nieta, consideró que parte de su bibliografía debería ser aprovechada como fuente de investigación para los interesados en conocer parte de la historia política, social, académica y educativa de esa región y cuál fue su protagonismo en el escenario nacional. Por eso motivo en 1996 ella decidió llevar estos materiales de investigación a la biblioteca del Centro Bartolomé de las Casas del Cusco.

Según sus familiares, los resultados en cuanto al cuidado de la tesis no fueron los adecuados en el Centro Bartolomé de las Casas del Cusco, en consecuencia, la Dra. Margarita Giesecke hizo las gestiones necesarias para trasladar esta bibliografía a la ciudad de Lima, siendo el Instituto Riva Agüero (IRA) elegido como la institución que reunía las condiciones logísticas para su cuidado y mantenimiento. Esta colección se encuentra temporalmente en el IRA, en calidad de depósito desde el 2003 y actualmente está a disposición de la comunidad académica.

Para hacer la consulta de la Colección del Dr. Giesecke, como nieta y familia directa me fue permitido realizar el reinventario de la misma en el año 2011 en el IRA, y cuenta con un tamaño relativamente pequeño de publicaciones, pero de gran valor para su dueño.

Las tesis ubicadas en los anaqueles que cobijan la biblioteca de la Colección del Dr. Giesecke, están empastadas con la finalidad de hacer pocos volúmenes, hallándose seis de siete volúmenes. Se pueden ordenar las ochenta y una tesis, teniendo en cuenta la temporalidad de las mismas, teniendo que la más antigua data de 1897 y no pertenece a la Universidad del Cusco, sino a la Universidad de Lima o San Marcos, cuyo autor es el Dr. Javier Prado y Ugarteche, titulada El porvenir de las razas en el Perú y las más actuales datan de 1943 y 1948, ambas de la Universidad del Cusco, la primera titulada Aspectos del arte incaico. Música y danza, y la segunda titulada Informe arqueológico de kollkce - wairachina, paucar cancha y atas - kcasa. También se pudo registrar una tesis sin fecha de sustentación, la cual titula El curso de las civilizaciones prehistóricas del Perú que pertenece al Dr. Félix Cosio.

La primera tesis sustentada en 1910, apenas asumía el cargo como rector, versó sobre Las industrias de los peruanos, en los periodos de su existencia, han seguido un movimiento proporcional a la evolución de la vida social y correspondió a Cosme Pacheco, donde examina la relación entre el potencial de los pueblos y su nivel de crecimiento productivo. La mayor parte de las tesis, se sitúan entre 1911 y 1922, salvo las tres antes mencionadas.

Se han contabilizado 81 tesis y de acuerdo al despliegue temático general se ha podido dar el siguiente orden para clasificarlas, tal como se muestra en el Cuadro 2:

Cuadro 2.

Clasificación temática de las tesis, Colección Giesecke

\section{1}

I. Matrimonio, divorcio, situación de mujer, familia.

II. Educación, diagnósticos de enseñanza.

III. Problema Indígena: Historia, cultura y arte indígena/ Organización y derecho indígena.

IV. Geología, Geografía y Economía del Cusco.

V. Desarrollo regional del Cusco.

VI. Derecho, Leyes y procedimientos jurídicos.

12
10
18
11
11
19


El ex rector, el Dr. Giesecke, fue profesor en la Facultad de Jurisprudencia y también en Ciencias Administrativas i Economía, además tuvo un notorio interés por la geología, geografía y recursos naturales, así como una gran predilección por la arqueología e historia Incas.

En el campo del conocimiento del Derecho, buscó promover la igualdad de derechos de la mujer y del indígena frente a la ley, habiendo en conjunto un total de 27 tesis que abarcan estas dos temáticas. Las que tuvieron un enfoque teórico democrático liberal, y buscaron fortalecer los fundamentos jurídicos peruanos para la defensa del derecho de la mujer y del indígena.

Por otro lado, y de manera complementaria las tesis sobre educación y desarrollo regional del Cusco, se centraron en la necesidad de la formación ciudadana a través de la educación y del desarrollo económico de todos los ciudadanos. Sobre un marcado individualismo jurídico y político, que debiera expresarse en la realización de deberes y derechos de las personas, al acceder a una escuela de calidad que implicara el contrato entre el Estado y la sociedad; por otro lado, los estudios sobre el derecho mismo y sus leyes, también reforzaron esta perspectiva, expresada en la tesis de Félix Cosío de 1916, titulada «Persona jurídica (ensayo de una clasificación)».

Por último y complementando las tesis sobre Desarrollo Regional del Cusco, los estudios sobre Geología, Geografía, Economía y Recursos Naturales del Cusco sirvieron para promover la mejora en la percepción económica y empresarial para el desarrollo, lo cual se vendría a complementar posteriormente con la creación de la Facultad de Agricultura en la década del cuarenta, aunque la fundamentación y solicitud de su creación se realizó por el rector Giesecke en el año 1922; en la creencia que ello permitiría que la agricultura sea realizada de manera científica y no empíricamente como se solía desenvolver por aquella época. A continuación, paso a analizar los datos obtenidos según la clasificación mencionada.

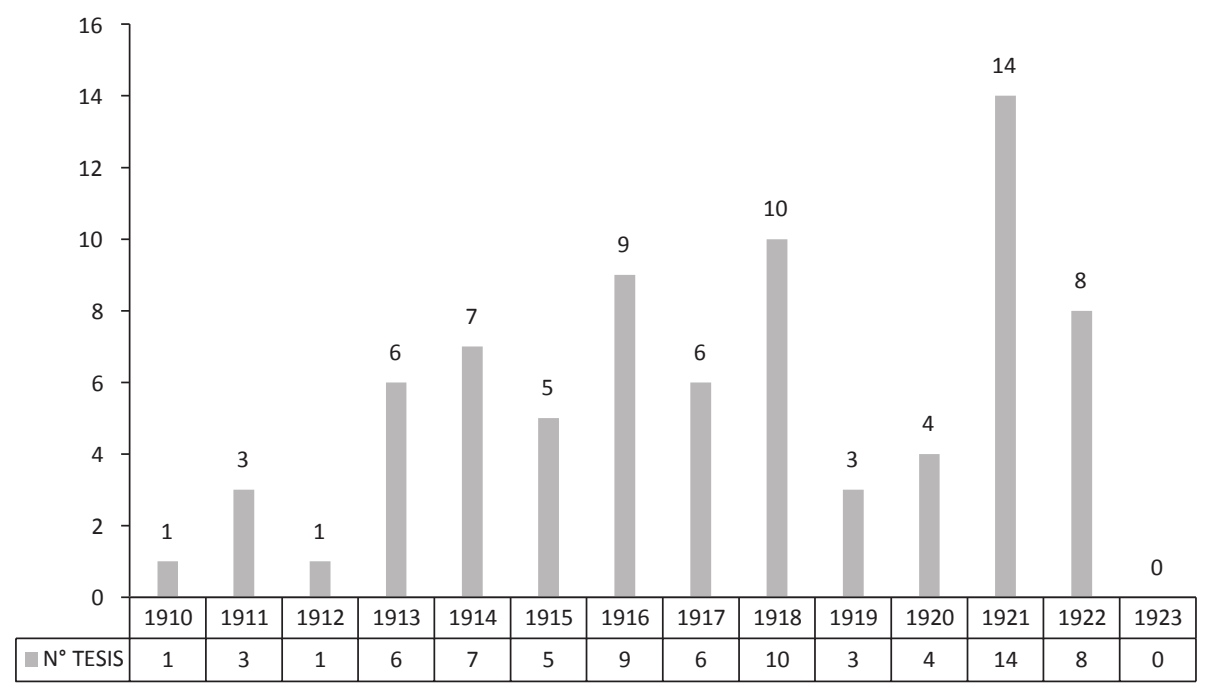

Figura 1. Tesis perteneciente a la colección Dr. Albert Giesecke durante su gestión como rector de la Universidad del Cusco (1910-1923)

La figura 1 muestra la tendencia que siguieron la producción de tesis durante la gestión del Dr. Giesecke. Durante sus más de 13 años de gestión, se pudo contabilizar 77 tesis distribuidos entre las especialidades que se impartía. Los años de mayor producción e investigación se dieron entre 1913 y 1922, donde se alcanzó una media de hasta 7 tesis sustentadas por año. El mayor pico de presentación de estos trabajos se dio en el año 1921, que coincidentemente se celebraba el centenario de la independencia peruana. Por lo visto se puede señalar que los estudiantes y la 
Universidad estaban decididos en participar de manera propositiva en el balance del desarrollo nacional luego de cien años como país independiente.

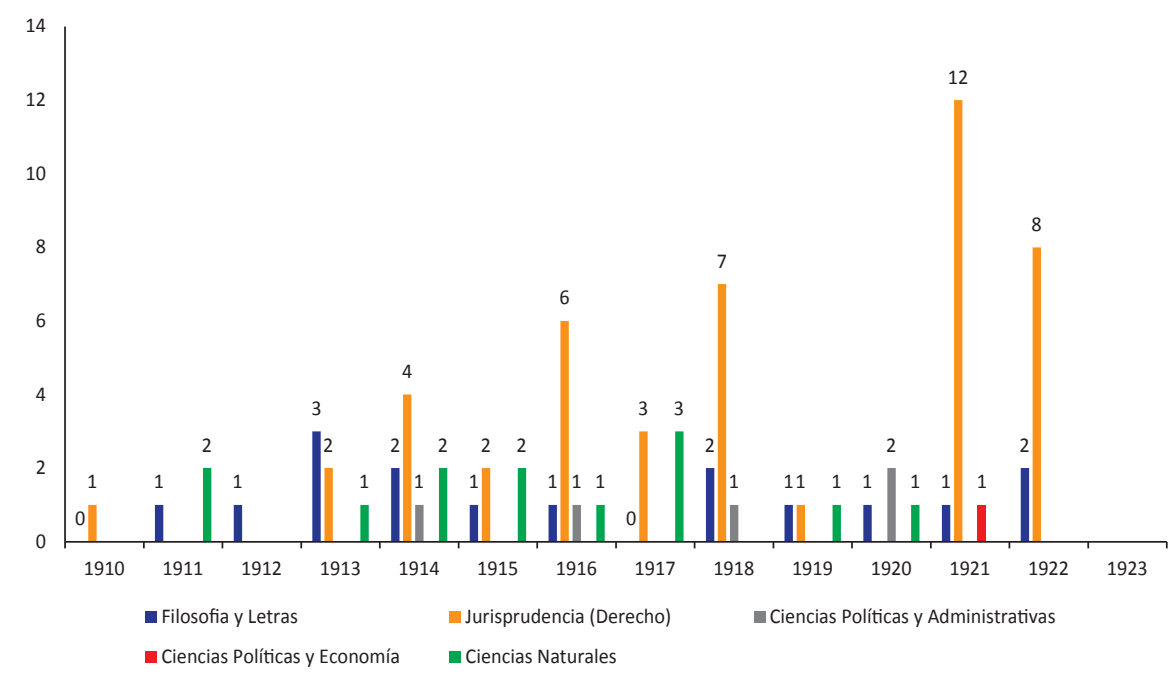

Figura 2. Tesis presentadas durante el rectorado del Dr. Albert Giesecke por especialidad (1910 - 1923)

En la figura 2 se muestra la producción de tesis presentadas por especialidad. Como se observa, la Facultad de Jurisprudencia es la que destaca sobre las demás, y desde la llegada de Giesecke sostuvo un crecimiento continuo. En total suman 46 las tesis solo en esta especialidad. Un dato a destacar es su crecimiento exponencial en el año 1921 que tiene su correlato en las celebraciones por el centenario del país. La segunda y tercera especialidad con más tesis eran la de filosofía y letras, ambas mantenían en promedio las dos terceras partes de las investigaciones en aquellos años.

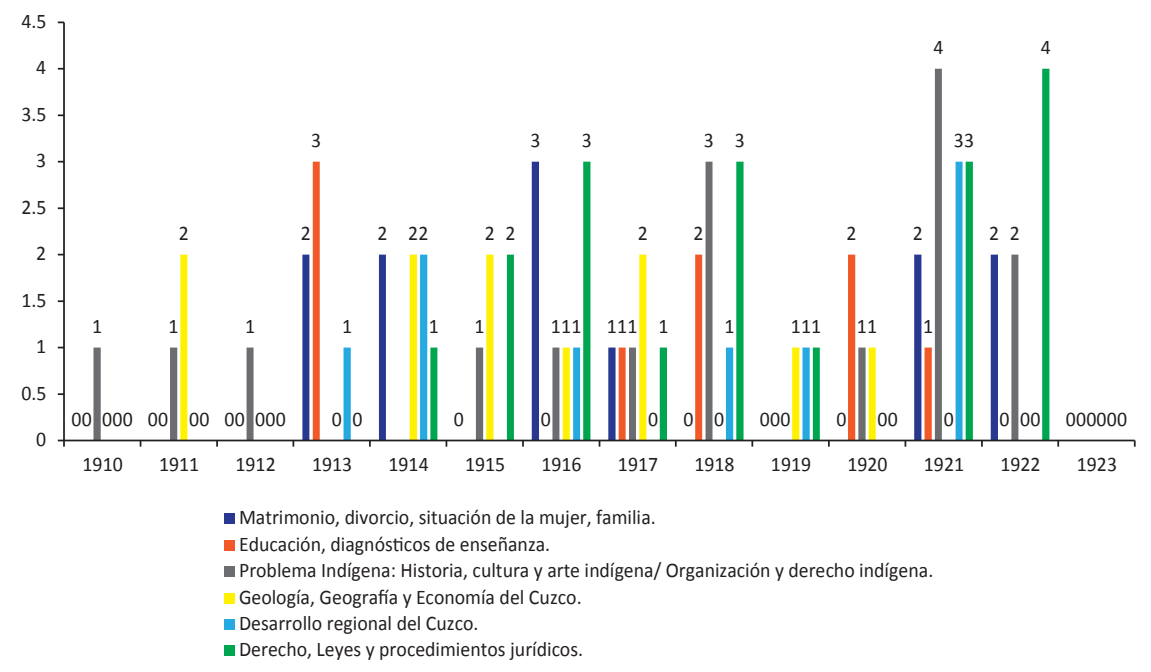

Figura 3. Producción de tesis por temas durante el rectorado del Dr. Albert Giesecke (Universidad del Cusco 1910 - 1923)

En la figura 3 se evidencia que la distribución temática fue proporcional y cada uno de ellas tuvo su año de mayor producción investigativa. Si bien el tema jurídico se registra recién a partir del año 1914, es la que alcanzó mayor cantidad publicaciones durante el rectorado de Giesecke. En segundo lugar, tenemos las investigaciones relacionadas al problema indígena, que implicaba el reconocimiento de su cultura, educación, organización y acceso a la tierra. Si sumamos estos 
dos factores, nos da un total de 36 tesis, que explicaría la relevancia de mantener en discusión constante el principal problema social de la región, a saber: el rol de lo jurídico en la organización social de la ciudad. Lo que se vino a denominar como el problema indígena y aparece en casi todos los años de su rectorado. Solo en 1923, donde no se ubicó ninguna tesis, dejó de presentarse este tema, porque concluyó su rectorado a mitad del año de 1923.

A diferencia de los temas mencionados, la cuestión educativa, geográfica, la organización familiar y la situación de la mujer se mantuvieron casi constantes. Mantuvieron una media de dos tesis por años. Según estas tesis, la educación, la familia y la situación en general no fue un tema relevante en los tres primeros años, recién en 1913 se empezó a tomar como área de interés. Asimismo, la geografía y economía del Cusco entre los años 1911 y 1917 la media se mantuvo constante, para luego ir descendiendo hasta desaparecer a partir de 1921. Finalmente, sobre lo relativo al desarrollo regional se visualiza que las investigaciones empezaron en 1913, puntualmente se presentaban una tesis al año y alcanzó un máximo de tres investigaciones en el año del centenario.

La cuantificación de estas tesis hace notar el propósito principal de su máxima autoridad, el cual era investigar la realidad general de toda la región y que los estudiantes vieran los problemas y posibles soluciones a partir de su propio entorno. La importancia de esta colección radica en que posee una vasta información de primer orden en base a los datos obtenidos de la realidad concreta. Su teoría parte del entorno para el entorno, basado en el enfoque pragmático de William James, donde la educación no era un solo conocer por conocer, sino, para enfrentar las contingencias de la vida cotidiana.

La historiografía peruana ha denominado República Aristocrática a los años que van desde 1895 a 1919, y donde según el consenso académico se ha situado como el nacimiento del estado moderno. Es dentro de este arco histórico donde trascurre gran parte de la gestión del Dr. Giesecke. Por su variada temática, estas tesis pueden ayudar a comprender mejor sobre los intereses y dinámicas propias de esta parte del sur del país, en un periodo de grandes cambios políticos, de crecimiento del aparato estatal y de demandas para las mejoras en el sector educativo a nivel nacional.

A continuación, muestro la Tabla 1 general de todos los títulos que tiene esta colección, donde 79 de ellas son para optar el grado de doctor y solo 2 para el grado de bachiller. También es importante resaltar las 2 primeras tesis de estudiantes mujeres en esta Universidad, en tiempos donde se pensaba que su labor se circunscribía al ámbito doméstico. La primera de ellas fue escrita por María Guadalupe Aguilar en 1916, titulada Bosquejo histórico y etnográfico de los pueblos de la América del sur. En este trabajo la autora describe el tipo de organización y tipo producción cultural de los principales pueblos sudamericanos, lo cual le permite comparar con las principales manifestaciones culturales de su región y sugerir la importancia de ellas para la identidad nacional.

Finalmente tenemos el trabajo de Elsa Pacheco Garmendia en 1943: Aspectos del arte incaico. Música y danza, en la cual describe las particularidades del folklore incaico y muestra cómo esta manifestación cultural se expresa en las ceremonias más importantes del Imperio incaico. La música como manifestación de la vida e historia de esta sociedad. Esta última tesis no corresponde a los años donde el Dr. Giesecke fue rector, pero al igual que la investigación de Javier Pardo en 1897, él guardó un ejemplar porque consideraba que estos temas deberían ser conocidos por las generaciones posteriores. 
Giesecke

\begin{tabular}{|c|c|c|c|c|}
\hline TESISTA & TITULO DE TESIS & TEMA DE INVESTIGACION & GRADO & UNIVERSIDAD / FACULTAD \\
\hline \multicolumn{5}{|l|}{1897} \\
\hline $\begin{array}{l}\text { Javier Prado y } \\
\text { Ugarteche }\end{array}$ & El porvenir de las razas en el Perú. & $\begin{array}{l}\text { Problema Indígena: Historia, } \\
\text { cultura y arte indígena/ } \\
\text { Organización y derecho } \\
\text { indígena }\end{array}$ & Doctor & $\begin{array}{l}\text { Jurisprudencia, Filosofía i } \\
\text { Letras } \\
\text { San Marcos }\end{array}$ \\
\hline \multicolumn{5}{|l|}{1910} \\
\hline Pacheco, Cosme & $\begin{array}{l}\text { Las industrias de los peruanos, } \\
\text { en los periodos de su existencia, } \\
\text { han seguido un movimiento } \\
\text { proporcional a la evolución de la } \\
\text { vida social. }\end{array}$ & Desarrollo regional del Cusco & Doctor & $\begin{array}{l}\text { Facultad de Jurisprudencia } \\
\text { San Marcos }\end{array}$ \\
\hline \multicolumn{5}{|l|}{1911} \\
\hline Corasao, Miguel & $\begin{array}{l}\text { Aprovechamiento de las aguas } \\
\text { selenitosas del Cusco. }\end{array}$ & $\begin{array}{l}\text { Geología, Geografía y } \\
\text { Economía del Cusco }\end{array}$ & Doctor & $\begin{array}{l}\text { Facultad Ciencias Naturales } \\
\text { San Antonio de Abad }\end{array}$ \\
\hline Fortunato Herrera & $\begin{array}{l}\text { Estudios geográficos en el } \\
\text { departamento. }\end{array}$ & $\begin{array}{l}\text { Geología, Geografía y } \\
\text { Economía del Cusco }\end{array}$ & Doctor & $\begin{array}{l}\text { Facultad de Ciencias Naturales } \\
\text { San Antonio de Abad }\end{array}$ \\
\hline García, J. Uriel & El arte incaico en el Cusco & $\begin{array}{l}\text { Problema Indígena: Historia, } \\
\text { cultura y arte indígena/ } \\
\text { Organización y derecho } \\
\text { indígena }\end{array}$ & Doctor & $\begin{array}{l}\text { Facultad de Filosofía i Letras } \\
\text { San Antonio de Abad }\end{array}$ \\
\hline \multicolumn{5}{|l|}{1912} \\
\hline $\begin{array}{l}\text { Valcárcel, Luis } \\
\text { Eduardo }\end{array}$ & Kon, Pachacamac, Uirakocha. & $\begin{array}{l}\text { Problema Indígena: Historia, } \\
\text { cultura y arte indígena/ } \\
\text { Organización y derecho } \\
\text { indígena }\end{array}$ & Bachiller & $\begin{array}{l}\text { Filosofía i Letras } \\
\text { San Antonio de Abad }\end{array}$ \\
\hline \multicolumn{5}{|l|}{1913} \\
\hline Casanova, Luis R. & Cusco a través de la historia. & Desarrollo regional del Cusco & Doctor & $\begin{array}{l}\text { Facultad de Letras i Filosofía } \\
\text { San Antonio de Abad }\end{array}$ \\
\hline $\begin{array}{l}\text { Jiménez Pacheco, } \\
\text { Julio }\end{array}$ & La mujer ante el derecho. & $\begin{array}{l}\text { Matrimonio, divorcio, } \\
\text { situación de mujer, familia }\end{array}$ & Doctor & $\begin{array}{l}\text { Facultad de Jurisprudencia } \\
\text { San Antonio de Abad }\end{array}$ \\
\hline $\begin{array}{l}\text { Monteagudo, } \\
\text { Federico }\end{array}$ & $\begin{array}{l}\text { La mujer casada debe disponer de } \\
\text { sus bienes, sin la autorización del } \\
\text { marido. }\end{array}$ & $\begin{array}{l}\text { Matrimonio, divorcio, } \\
\text { situación de mujer, familia }\end{array}$ & Doctor & $\begin{array}{l}\text { Facultad de Jurisprudencia } \\
\text { San Antonio de Abad }\end{array}$ \\
\hline Pareja Cristóbal & La disciplina escolar en el Cusco. & $\begin{array}{l}\text { Educación, diagnósticos, } \\
\text { enseñanza }\end{array}$ & Doctor & $\begin{array}{l}\text { Facultad de Letras I Filosofía } \\
\text { San Antonio de Abad }\end{array}$ \\
\hline $\begin{array}{l}\text { Valcárcel, Luis } \\
\text { Eduardo }\end{array}$ & $\begin{array}{l}\text { La universidad del Cusco su nueva } \\
\text { organización. }\end{array}$ & $\begin{array}{l}\text { Educación, diagnósticos, } \\
\text { enseñanza }\end{array}$ & Doctor & $\begin{array}{l}\text { Letras I Filosofía San Antonio } \\
\text { de Abad }\end{array}$ \\
\hline Luna, Humberto & Paidología (el niño del Cusco). & $\begin{array}{l}\text { Educación, diagnósticos, } \\
\text { enseñanza }\end{array}$ & Bachiller & $\begin{array}{l}\text { Facultad de Ciencias Naturales } \\
\text { San Antonio de Abad }\end{array}$ \\
\hline \multicolumn{5}{|l|}{1914} \\
\hline Ismodes, Emilio & $\begin{array}{l}\text { El divorcio es conforme al derecho } \\
\text { natural y una exigencia natural en la } \\
\text { actualidad. }\end{array}$ & $\begin{array}{l}\text { Matrimonio, divorcio, } \\
\text { situación de mujer, familia }\end{array}$ & Doctor & $\begin{array}{l}\text { Facultad de Jurisprudencia } \\
\text { San Antonio de Abad }\end{array}$ \\
\hline $\begin{array}{l}\text { Cáceres, Juan } \\
\text { Nicolás }\end{array}$ & $\begin{array}{l}\text { La ley debe considerar el } \\
\text { matrimonio, solo desde el punto } \\
\text { de vista de sus afectos civiles e } \\
\text { independiente a las creencias } \\
\text { religiosas de los contrayentes. }\end{array}$ & $\begin{array}{l}\text { Matrimonio, divorcio, } \\
\text { situación de mujer, familia }\end{array}$ & Doctor & $\begin{array}{l}\text { Facultad de Jurisprudencia } \\
\text { San Antonio de Abad }\end{array}$ \\
\hline $\begin{array}{l}\text { Arguedas, Luis } \\
\text { Alberto }\end{array}$ & $\begin{array}{l}\text { Plasmogenia y estructuras } \\
\text { artificiales. }\end{array}$ & $\begin{array}{l}\text { Geología, Geografía y } \\
\text { Economía del Cusco }\end{array}$ & Doctor & $\begin{array}{l}\text { Facultad de Ciencias Naturales } \\
\text { San Antonio de Abad }\end{array}$ \\
\hline Velazco, Andrés & Estudio de la coca (eritróxilon coca). & $\begin{array}{l}\text { Geología, Geografía y } \\
\text { Economía del Cusco }\end{array}$ & Doctor & $\begin{array}{l}\text { Facultad de Ciencias Naturales } \\
\text { San Antonio de Abad }\end{array}$ \\
\hline
\end{tabular}


Colección de tesis de la Universidad del Cusco sustentadas en el rectorado del Dr. Albert A. Giesecke (1910-1923)

\begin{tabular}{|c|c|c|c|c|}
\hline TESISTA & TITULO DE TESIS & TEMA DE INVESTIGACION & GRADO & UNIVERSIDAD / FACULTAD \\
\hline $\begin{array}{l}\text { Valcárcel, Luis } \\
\text { Eduardo }\end{array}$ & La cuestión agraria en el Cusco. & Desarrollo regional del Cusco & Bachiller & $\begin{array}{l}\text { Facultad Ciencias Políticas i } \\
\text { Administrativas San Antonio } \\
\text { de Abad }\end{array}$ \\
\hline $\begin{array}{l}\text { Flores Fernández, } \\
\text { Leoncio }\end{array}$ & $\begin{array}{l}\text { Las minas deben corresponder al } \\
\text { propietario del terreno donde se } \\
\text { halla. }\end{array}$ & Desarrollo regional del Cusco & Doctor & $\begin{array}{l}\text { Facultad de Jurisprudencia } \\
\text { San Antonio de Abad }\end{array}$ \\
\hline $\begin{array}{l}\text { Gabino Ugarte, } \\
\text { Jorge }\end{array}$ & $\begin{array}{l}\text { El derecho civil i su influencia en la } \\
\text { criminalidad. }\end{array}$ & $\begin{array}{l}\text { Derecho, Leyes y } \\
\text { procedimientos jurídicos }\end{array}$ & Doctor & $\begin{array}{l}\text { Facultad de Jurisprudencia } \\
\text { San Antonio de Abad }\end{array}$ \\
\hline \multicolumn{5}{|l|}{1915} \\
\hline Rodríguez, Jesús & $\begin{array}{l}\text { Estudio del contrato de enganche: } \\
\text { sus funestas consecuencias y } \\
\text { sistema que debe preferirse }\end{array}$ & $\begin{array}{l}\text { Problema Indígena: Historia, } \\
\text { cultura y arte indígena/ } \\
\text { Organización y derecho } \\
\text { indígena }\end{array}$ & Doctor & $\begin{array}{l}\text { Facultad de Letras i Filosofía } \\
\text { San Antonio de Abad }\end{array}$ \\
\hline Leandro, Pareja & $\begin{array}{l}\text { Descubrimiento de un reactivo } \\
\text { indicador. }\end{array}$ & $\begin{array}{l}\text { Geología, Geografía y } \\
\text { Economía del Cusco }\end{array}$ & Doctor & $\begin{array}{l}\text { Facultad de Ciencias Naturales } \\
\text { San Antonio de Abad }\end{array}$ \\
\hline Luna, Humberto & Oecodoma cephalotes (cuqui). & $\begin{array}{l}\text { Geología, Geografía y } \\
\text { Economía del Cusco }\end{array}$ & Doctor & $\begin{array}{l}\text { Facultad de Ciencias Naturales } \\
\text { San Antonio de Abad }\end{array}$ \\
\hline Bernizon, Juan P. & $\begin{array}{l}\text { Inmunidades de los agentes } \\
\text { diplomáticos. }\end{array}$ & $\begin{array}{l}\text { Derecho, Leyes y } \\
\text { procedimientos jurídicos }\end{array}$ & Doctor & $\begin{array}{l}\text { Facultad de Jurisprudencia } \\
\text { San Antonio de Abad }\end{array}$ \\
\hline $\begin{array}{l}\text { Serafín Meneses, } \\
\text { Carlos }\end{array}$ & $\begin{array}{l}\text { Los juicios de imprenta deben } \\
\text { pertenecer, únicamente, al fuero } \\
\text { común. }\end{array}$ & $\begin{array}{l}\text { Derecho, Leyes y } \\
\text { procedimientos jurídicos }\end{array}$ & Doctor & $\begin{array}{l}\text { Facultad de Jurisprudencia } \\
\text { San Antonio de Abad }\end{array}$ \\
\hline \multicolumn{5}{|l|}{1916} \\
\hline $\begin{array}{l}\text { Manchego Muñoz, } \\
\text { Teodorico }\end{array}$ & $\begin{array}{l}\text { Necesarias reformas de la adopción } \\
\text { en la legislación patria. }\end{array}$ & $\begin{array}{l}\text { Matrimonio, divorcio, } \\
\text { situación de mujer, familia }\end{array}$ & Doctor & $\begin{array}{l}\text { Facultad de Jurisprudencia } \\
\text { San Antonio de Abad }\end{array}$ \\
\hline R. Casanova, Luis & $\begin{array}{l}\text { La familia en el Perú i su evolución } \\
\text { histórica. }\end{array}$ & $\begin{array}{l}\text { Matrimonio, divorcio, } \\
\text { situación de mujer, familia }\end{array}$ & Doctor & $\begin{array}{l}\text { Facultad de Jurisprudencia } \\
\text { San Antonio de Abad }\end{array}$ \\
\hline $\begin{array}{l}\text { Modesto } \\
\text { Villanueva, Víctor }\end{array}$ & Derechos de los hijos ilegítimos. & $\begin{array}{l}\text { Matrimonio, divorcio, } \\
\text { situación de mujer, familia }\end{array}$ & Doctor & $\begin{array}{l}\text { Facultad de Jurisprudencia } \\
\text { San Antonio de Abad }\end{array}$ \\
\hline $\begin{array}{l}\text { Aguilar, María } \\
\text { Guadalupe }\end{array}$ & $\begin{array}{l}\text { Bosquejo histórico y etnográfico de } \\
\text { los pueblos de la América del sur. }\end{array}$ & $\begin{array}{l}\text { Problema Indígena: Historia, } \\
\text { cultura y arte indígena/ } \\
\text { Organización y derecho } \\
\text { indígena }\end{array}$ & Doctor & $\begin{array}{l}\text { Facultad de Filosofía i Letras } \\
\text { San Antonio de Abad }\end{array}$ \\
\hline Honorio Pareja, G. & El petróleo hai en el departamento & $\begin{array}{l}\text { Geología, Geografía y } \\
\text { Economía del Cusco }\end{array}$ & Doctor & $\begin{array}{l}\text { Facultad de Ciencias Naturales } \\
\text { San Antonio de Abad }\end{array}$ \\
\hline Rosell, Enrique & $\begin{array}{l}\text { Monografías de la provincia de la } \\
\text { convención }\end{array}$ & Desarrollo regional del Cusco & Doctor & $\begin{array}{l}\text { Facultad Ciencias Políticas i } \\
\text { Administrativas San Antonio } \\
\text { de Abad }\end{array}$ \\
\hline $\begin{array}{l}\text { Manuel Chávez } \\
\text { Fernández }\end{array}$ & $\begin{array}{l}\text { La descentralización i reforma } \\
\text { judicial. }\end{array}$ & $\begin{array}{l}\text { Derecho, Leyes y } \\
\text { procedimientos jurídicos }\end{array}$ & Doctor & $\begin{array}{l}\text { Facultad de Jurisprudencia } \\
\text { San Antonio de Abad }\end{array}$ \\
\hline $\begin{array}{l}\text { Alicante Esteban, } \\
\text { M. }\end{array}$ & $\begin{array}{l}\text { La prescripción de la acción penal } \\
\text { durante el sumario. }\end{array}$ & $\begin{array}{l}\text { Derecho, Leyes y } \\
\text { procedimientos jurídicos }\end{array}$ & Doctor & $\begin{array}{l}\text { Facultad de Jurisprudencia } \\
\text { San Antonio de Abad }\end{array}$ \\
\hline Cosio, Félix & $\begin{array}{l}\text { Persona jurídica (ensayo de una } \\
\text { clasificación). }\end{array}$ & $\begin{array}{l}\text { Derecho, Leyes y } \\
\text { procedimientos jurídicos }\end{array}$ & Doctor & $\begin{array}{l}\text { Facultad de Jurisprudencia } \\
\text { San Antonio de Abad }\end{array}$ \\
\hline \multicolumn{5}{|l|}{1917} \\
\hline Coello, Alejandro & $\begin{array}{l}\text { La estereoquímica y sus nuevas } \\
\text { orientaciones en orden a la } \\
\text { evolución de la enseñanza de } \\
\text { química orgánica. }\end{array}$ & $\begin{array}{l}\text { Educación, diagnósticos, } \\
\text { enseñanza }\end{array}$ & Doctor & $\begin{array}{l}\text { Facultad de Ciencias Naturales } \\
\text { San Antonio de Abad }\end{array}$ \\
\hline
\end{tabular}


Giesecke

\begin{tabular}{|c|c|c|c|c|}
\hline TESISTA & TITULO DE TESIS & TEMA DE INVESTIGACION & GRADO & UNIVERSIDAD / FACULTAD \\
\hline $\begin{array}{l}\text { Pacheco Delgado, } \\
\text { Jerónimo }\end{array}$ & $\begin{array}{l}\text { Las prisiones en el Perú no cumplen } \\
\text { el fin jurídico que la pena se } \\
\text { propone. En su aspecto moral, } \\
\text { material y social. Estudio analítico } \\
\text { del departamento del Cusco y } \\
\text { provincias de este. La penitenciaria } \\
\text { de Lima y algunas cárceles más. } \\
\text { Sistema que sería apropiado para la } \\
\text { formación de prisiones en el Perú. } \\
\text { Plan y desarrollo de este. Proyecto } \\
\text { de ley que debiera reglamentarla. }\end{array}$ & $\begin{array}{l}\text { Problema Indígena: Historia, } \\
\text { cultura y arte indígena/ } \\
\text { Organización y derecho } \\
\text { indígena }\end{array}$ & Doctor & $\begin{array}{l}\text { Facultad de Jurisprudencia } \\
\text { San Antonio de Abad }\end{array}$ \\
\hline $\begin{array}{l}\text { Alarcón, } \\
\text { Alejandrina }\end{array}$ & $\begin{array}{l}\text { Acción de los glaciares en el valle } \\
\text { del Cusco. }\end{array}$ & $\begin{array}{l}\text { Geología, Geografía y } \\
\text { Economía del Cusco }\end{array}$ & Doctor & $\begin{array}{l}\text { Facultad de Ciencias Naturales } \\
\text { San Antonio de Abad }\end{array}$ \\
\hline Corasao, Demetrio & $\begin{array}{l}\text { Responsabilidad de los peritos y } \\
\text { testigos i el tiempo en que debe } \\
\text { entablarse la acción de falsa } \\
\text { exposición pericial o de falso } \\
\text { testimonio en las causas penales. }\end{array}$ & $\begin{array}{l}\text { Derecho, Leyes y } \\
\text { procedimientos jurídicos }\end{array}$ & Doctor & $\begin{array}{l}\text { Facultad de Jurisprudencia } \\
\text { San Antonio de Abad }\end{array}$ \\
\hline $\begin{array}{l}\text { Ponce De León } \\
\text { Federico }\end{array}$ & $\begin{array}{l}\text { Herbert E. Gregory y la geología del } \\
\text { Cusco. }\end{array}$ & $\begin{array}{l}\text { Geología, Geografía y } \\
\text { Economía del Cusco }\end{array}$ & Doctor & $\begin{array}{l}\text { Facultad de Ciencias Naturales } \\
\text { San Antonio de Abad }\end{array}$ \\
\hline $\begin{array}{l}\text { Valencia Pinto, M. } \\
\text { Ismael }\end{array}$ & La sesión de los cónyuges. & $\begin{array}{l}\text { Matrimonio, divorcio, } \\
\text { situación de mujer, familia }\end{array}$ & Doctor & $\begin{array}{l}\text { Facultad de Jurisprudencia } \\
\text { San Antonio de Abad }\end{array}$ \\
\hline \multicolumn{5}{|l|}{1918} \\
\hline $\begin{array}{l}\text { José Luis } \\
\text { Bustamante y } \\
\text { Rivero }\end{array}$ & La crisis universitaria & $\begin{array}{l}\text { Educación, diagnósticos, } \\
\text { enseñanza }\end{array}$ & Doctor & $\begin{array}{l}\text { Facultad de Filosofía i Letras } \\
\text { San Antonio de Abad }\end{array}$ \\
\hline $\begin{array}{l}\text { Ponce De León, } \\
\text { Francisco }\end{array}$ & $\begin{array}{l}\text { Aspectos económicos del problema } \\
\text { indígena. }\end{array}$ & $\begin{array}{l}\text { Problema Indígena: Historia, } \\
\text { cultura y arte indígena/ } \\
\text { Organización y derecho } \\
\text { indígena }\end{array}$ & Doctor & $\begin{array}{l}\text { Facultad de Jurisprudencia } \\
\text { San Antonio de Abad }\end{array}$ \\
\hline $\begin{array}{l}\text { Garrido Mendivil, } \\
\text { J.M. }\end{array}$ & El hebradgo. & $\begin{array}{l}\text { Problema Indígena: Historia, } \\
\text { cultura y arte indígena/ } \\
\text { Organización y derecho } \\
\text { indígena }\end{array}$ & Doctor & $\begin{array}{l}\text { Facultad de Jurisprudencia } \\
\text { San Antonio de Abad }\end{array}$ \\
\hline Bravo Mejía, G. & $\begin{array}{l}\text { Organizaciones de las escuelas } \\
\text { rurales }\end{array}$ & $\begin{array}{l}\text { Educación, diagnósticos, } \\
\text { enseñanza }\end{array}$ & Doctor & $\begin{array}{l}\text { Facultad de Filosofía i Letras } \\
\text { San Antonio de Abad. }\end{array}$ \\
\hline Ordoñez, Pedro & $\begin{array}{l}\text { Los varayocs, estudio de una forma } \\
\text { de gobierno y administración local. }\end{array}$ & $\begin{array}{l}\text { Problema Indígena: Historia, } \\
\text { cultura y arte indígena/ } \\
\text { Organización y derecho } \\
\text { indígena }\end{array}$ & Doctor & $\begin{array}{l}\text { Facultad de Jurisprudencia } \\
\text { San Antonio de Abad }\end{array}$ \\
\hline Dávila Peña, Julio & $\begin{array}{l}\text { El juramento y sus clases en el } \\
\text { derecho moderno. }\end{array}$ & $\begin{array}{l}\text { Derecho, Leyes y } \\
\text { procedimientos jurídicos }\end{array}$ & Doctor & $\begin{array}{l}\text { Facultad de Jurisprudencia } \\
\text { San Antonio de Abad }\end{array}$ \\
\hline Pareja S., Cristóbal & $\begin{array}{l}\text { Necesidad de reformar la } \\
\text { clasificación de las formas de los } \\
\text { testamentos, instituyendo, además, } \\
\text { el testamento ológrafo. }\end{array}$ & $\begin{array}{l}\text { Derecho, Leyes y } \\
\text { procedimientos jurídicos }\end{array}$ & Doctor & $\begin{array}{l}\text { Facultad de Jurisprudencia } \\
\text { San Antonio de Abad }\end{array}$ \\
\hline $\begin{array}{l}\text { Flores Ayala, } \\
\text { Timoteo }\end{array}$ & $\begin{array}{l}\text { La autonomía de las } \\
\text { municipalidades es indispensable } \\
\text { para la realización del ideal } \\
\text { regionalista en el Perú. }\end{array}$ & Desarrollo regional del Cusco & Doctor & $\begin{array}{l}\text { Facultad Ciencias Políticas i } \\
\text { Administrativas San Antonio } \\
\text { de Abad }\end{array}$ \\
\hline Vera Rebollar, Luis & $\begin{array}{l}\text { El divorcio absoluto ante la } \\
\text { legislación peruana }\end{array}$ & $\begin{array}{l}\text { Derecho, Leyes y } \\
\text { procedimientos jurídicos }\end{array}$ & Doctor & $\begin{array}{l}\text { Facultad de Jurisprudencia } \\
\text { San Antonio de Abad }\end{array}$ \\
\hline Aguilar, Rafael & Libertad testamentaria. & $\begin{array}{l}\text { Derecho, Leyes y } \\
\text { procedimientos jurídicos }\end{array}$ & Doctor & $\begin{array}{l}\text { Facultad de Jurisprudencia } \\
\text { San Antonio de Abad }\end{array}$ \\
\hline
\end{tabular}


Colección de tesis de la Universidad del Cusco sustentadas en el rectorado del Dr. Albert A. Giesecke (1910-1923)

\begin{tabular}{|c|c|c|c|c|}
\hline TESISTA & TITULO DE TESIS & TEMA DE INVESTIGACION & GRADO & UNIVERSIDAD / FACULTAD \\
\hline \multicolumn{5}{|l|}{1919} \\
\hline Moscoso, J. L. & $\begin{array}{l}\text { La muerte es una } \\
\text { consecuencia necesaria para el } \\
\text { perfeccionamiento del organismo. }\end{array}$ & $\begin{array}{l}\text { Geología, Geografía y } \\
\text { Economía del Cusco }\end{array}$ & Doctor & $\begin{array}{l}\text { Facultad de Ciencias Naturales } \\
\text { San Antonio de Abad }\end{array}$ \\
\hline Gibaja, Mariano C. & $\begin{array}{l}\text { Influencia de la iglesia en la historia } \\
\text { del Cusco. }\end{array}$ & Desarrollo regional del Cusco & Doctor & $\begin{array}{l}\text { Facultad de Filosofía i Letras } \\
\text { San Antonio de Abad }\end{array}$ \\
\hline Luna, Humberto & Observaciones criminológicas. & $\begin{array}{l}\text { Derecho, Leyes y } \\
\text { procedimientos jurídicos }\end{array}$ & Doctor & $\begin{array}{l}\text { Facultad de Jurisprudencia } \\
\text { San Antonio de Abad }\end{array}$ \\
\hline \multicolumn{5}{|l|}{1920} \\
\hline Bravo Mejía, G. & $\begin{array}{l}\text { La reforma en los estudios de la } \\
\text { facultad de filosofía y letras. }\end{array}$ & $\begin{array}{l}\text { Educación, diagnósticos, } \\
\text { enseñanza }\end{array}$ & Doctor & $\begin{array}{l}\text { Ciencias Políticas i } \\
\text { administrativas Universidad } \\
\text { San Agustín de Arequipa }\end{array}$ \\
\hline Aguilar, Rafael & El Estado y la enseñanza. & $\begin{array}{l}\text { Educación, diagnósticos, } \\
\text { enseñanza }\end{array}$ & Doctor & $\begin{array}{l}\text { Facultad Ciencias Políticas i } \\
\text { Administrativas San Antonio } \\
\text { de Abad }\end{array}$ \\
\hline Límaco, Eladio & Filosofía kechua. & $\begin{array}{l}\text { Problema Indígena: Historia, } \\
\text { cultura y arte indígena/ } \\
\text { Organización y derecho } \\
\text { indígena }\end{array}$ & Doctor & $\begin{array}{l}\text { Facultad de Filosofía i Letras } \\
\text { San Antonio de Abad }\end{array}$ \\
\hline $\begin{array}{l}\text { Castro, } \\
\text { Humberto José }\end{array}$ & $\begin{array}{l}\text { Nueva teoría para explicar el } \\
\text { mecanismo de los fenómenos } \\
\text { catalíticos y la fermentación. }\end{array}$ & $\begin{array}{l}\text { Geología, Geografía y } \\
\text { Economía del Cusco }\end{array}$ & Doctor & $\begin{array}{l}\text { Facultad de Ciencias Naturales } \\
\text { San Antonio de Abad }\end{array}$ \\
\hline \multicolumn{5}{|l|}{1921} \\
\hline Huarcaya, Mateo & Matrimonio civil y divorcio. & $\begin{array}{l}\text { Matrimonio, divorcio, } \\
\text { situación de mujer, familia }\end{array}$ & Doctor & $\begin{array}{l}\text { Facultad de Jurisprudencia } \\
\text { San Antonio de Abad }\end{array}$ \\
\hline Ponce De León & Comentarios a la ley de divorcio. & $\begin{array}{l}\text { Matrimonio, divorcio, } \\
\text { situación de mujer, familia }\end{array}$ & Doctor & $\begin{array}{l}\text { Facultad de Jurisprudencia } \\
\text { San Antonio de Abad }\end{array}$ \\
\hline $\begin{array}{l}\text { Miranda, Julio } \\
\text { Cesar }\end{array}$ & $\begin{array}{l}\text { Locales escolares del Cusco } \\
\text { (homenaje de pedagogía aplicada a } \\
\text { mis compañeros de labor). }\end{array}$ & $\begin{array}{l}\text { Educación, diagnósticos, } \\
\text { enseñanza }\end{array}$ & Doctor & $\begin{array}{l}\text { Facultad de Filosofía i Letras } \\
\text { San Antonio de Abad }\end{array}$ \\
\hline Ochoa G., Luis/ & $\begin{array}{l}\text { El problema indígena y el código de } \\
\text { procedimientos civiles. }\end{array}$ & $\begin{array}{l}\text { Problema Indígena: Historia, } \\
\text { cultura y arte indígena/ } \\
\text { Organización y derecho } \\
\text { indígena }\end{array}$ & Doctor & $\begin{array}{l}\text { Facultad de Jurisprudencia } \\
\text { San Antonio de Abad }\end{array}$ \\
\hline $\begin{array}{l}\text { Gonzales Gamarra, } \\
\text { Luis F. }\end{array}$ & $\begin{array}{l}\text { Criminalidad indígena. Colonias } \\
\text { penales agrícolas. }\end{array}$ & $\begin{array}{l}\text { Problema Indígena: Historia, } \\
\text { cultura y arte indígena/ } \\
\text { Organización y derecho } \\
\text { indígena }\end{array}$ & Doctor & $\begin{array}{l}\text { Facultad de Jurisprudencia } \\
\text { San Antonio de Abad }\end{array}$ \\
\hline Aguilar, Luis F. & La criminalidad indígena. & $\begin{array}{l}\text { Problema Indígena: Historia, } \\
\text { cultura y arte indígena/ } \\
\text { Organización y derecho } \\
\text { indígena }\end{array}$ & Doctor & $\begin{array}{l}\text { Facultad de Jurisprudencia } \\
\text { San Antonio de Abad }\end{array}$ \\
\hline Guillén, Víctor M. & $\begin{array}{l}\text { La reintegración de la propiedad } \\
\text { comunal indígena. }\end{array}$ & $\begin{array}{l}\text { Problema Indígena: Historia, } \\
\text { cultura y arte indígena/ } \\
\text { Organización y derecho } \\
\text { indígena }\end{array}$ & Doctor & $\begin{array}{l}\text { Facultad de Jurisprudencia } \\
\text { San Antonio de Abad }\end{array}$ \\
\hline Ferro, José Ignacio & $\begin{array}{l}\text { La provincia del Cusco i el distrito } \\
\text { de Oropesa. }\end{array}$ & Desarrollo regional del Cusco & Doctor & $\begin{array}{l}\text { Facultad de Jurisprudencia } \\
\text { San Antonio de Abad }\end{array}$ \\
\hline Roca, Gerardo J. & $\begin{array}{l}\text { Estudio económico de la provincia } \\
\text { del Cusco. }\end{array}$ & Desarrollo regional del Cusco & Doctor & $\begin{array}{l}\text { Facultad de Jurisprudencia } \\
\text { San Antonio de Abad }\end{array}$ \\
\hline Límaco, Carlos E. & El regionalismo. & Desarrollo regional del Cusco & Doctor & $\begin{array}{l}\text { Facultad Ciencias Políticas i } \\
\text { Economía San Antonio de } \\
\text { Abad }\end{array}$ \\
\hline
\end{tabular}


Giesecke

\begin{tabular}{|c|c|c|c|c|}
\hline TESISTA & TITULO DE TESIS & TEMA DE INVESTIGACION & GRADO & UNIVERSIDAD / FACULTAD \\
\hline $\begin{array}{l}\text { Usandivaras, } \\
\text { Octavio }\end{array}$ & $\begin{array}{l}\text { Concepto jurídico de bienes } \\
\text { inmuebles para el efecto de la } \\
\text { hipoteca i su inscripción en el } \\
\text { registro de la propiedad. }\end{array}$ & $\begin{array}{l}\text { Derecho, Leyes y } \\
\text { procedimientos jurídicos }\end{array}$ & Doctor & $\begin{array}{l}\text { Facultad de Jurisprudencia } \\
\text { San Antonio de Abad }\end{array}$ \\
\hline $\begin{array}{l}\text { Delgado De La } \\
\text { Flor, Rodrigo }\end{array}$ & La acción penal. & $\begin{array}{l}\text { Derecho, Leyes y } \\
\text { procedimientos jurídicos }\end{array}$ & Doctor & $\begin{array}{l}\text { Facultad de Jurisprudencia } \\
\text { San Antonio de Abad }\end{array}$ \\
\hline $\begin{array}{l}\text { Gallegos, Cesar } \\
\text { Jesús }\end{array}$ & Restablecimiento de la querella. & $\begin{array}{l}\text { Derecho, Leyes y } \\
\text { procedimientos jurídicos }\end{array}$ & Doctor & $\begin{array}{l}\text { Facultad de Jurisprudencia } \\
\text { San Antonio de Abad }\end{array}$ \\
\hline $\begin{array}{l}\text { Cáceres Gaudet, } \\
\text { Guillermo }\end{array}$ & $\begin{array}{l}\text { La cedula hipotecaria como } \\
\text { exponente del crédito territorial en } \\
\text { el Perú. }\end{array}$ & Desarrollo regional del Cusco & Doctor & $\begin{array}{l}\text { Facultad de Jurisprudencia } \\
\text { San Antonio de Abad }\end{array}$ \\
\hline \multicolumn{5}{|l|}{1922} \\
\hline García, J. Mariano & $\begin{array}{l}\text { La capacidad jurídica de la mujer } \\
\text { casada, debe ampliarse en nuestra } \\
\text { legislación civil. }\end{array}$ & $\begin{array}{l}\text { Matrimonio, divorcio, } \\
\text { situación de mujer, familia }\end{array}$ & Doctor & $\begin{array}{l}\text { Facultad de Jurisprudencia } \\
\text { San Antonio de Abad }\end{array}$ \\
\hline $\begin{array}{l}\text { Casafranca, } \\
\text { Manuel }\end{array}$ & $\begin{array}{l}\text { Derechos de los hijos nacidos fuera } \\
\text { de matrimonio. }\end{array}$ & $\begin{array}{l}\text { Matrimonio, divorcio, } \\
\text { situación de mujer, familia }\end{array}$ & Doctor & $\begin{array}{l}\text { Facultad de Jurisprudencia } \\
\text { San Antonio de Abad }\end{array}$ \\
\hline Gibaja, Ruperto & $\begin{array}{l}\text { Reorganización de la propiedad } \\
\text { indígena. }\end{array}$ & $\begin{array}{l}\text { Problema Indígena: Historia, } \\
\text { cultura y arte indígena/ } \\
\text { Organización y derecho } \\
\text { indígena }\end{array}$ & Doctor & $\begin{array}{l}\text { Facultad de Jurisprudencia } \\
\text { San Antonio de Abad }\end{array}$ \\
\hline Ochoa, A. Justo & Colonias penales. & $\begin{array}{l}\text { Problema Indígena: Historia, } \\
\text { cultura y arte indígena/ } \\
\text { Organización y derecho } \\
\text { indígena }\end{array}$ & Doctor & $\begin{array}{l}\text { Facultad de Jurisprudencia } \\
\text { San Antonio de Abad }\end{array}$ \\
\hline $\begin{array}{l}\text { Estrada Galdo, } \\
\text { Luis A. }\end{array}$ & $\begin{array}{l}\text { Las ventajas del procedimiento } \\
\text { penal, del novísimo código en } \\
\text { vigencia, en la profilaxis criminal. El } \\
\text { voto de consecuencia. }\end{array}$ & $\begin{array}{l}\text { Derecho, Leyes y } \\
\text { procedimientos jurídicos }\end{array}$ & Doctor & $\begin{array}{l}\text { Facultad de Jurisprudencia } \\
\text { San Antonio de Abad }\end{array}$ \\
\hline Salas Alberto A. & La vagancia como factor del crimen. & $\begin{array}{l}\text { Derecho, Leyes y } \\
\text { procedimientos jurídicos }\end{array}$ & Doctor & $\begin{array}{l}\text { Facultad de Jurisprudencia } \\
\text { San Antonio de Abad }\end{array}$ \\
\hline $\begin{array}{l}\text { Mariscal, } \\
\text { Alejandro }\end{array}$ & $\begin{array}{l}\text { La delincuencia precoz y su } \\
\text { penalidad. }\end{array}$ & $\begin{array}{l}\text { Derecho, Leyes y } \\
\text { procedimientos jurídicos }\end{array}$ & Doctor & $\begin{array}{l}\text { Facultad de Jurisprudencia } \\
\text { San Antonio de Abad }\end{array}$ \\
\hline Bueno, Manuel & $\begin{array}{l}\text { El comprador de una herencia, no } \\
\text { puede, según nuestra legislación, } \\
\text { pedir la nulidad del testamento, que } \\
\text { sirve de título a su vendedor. }\end{array}$ & $\begin{array}{l}\text { Derecho, Leyes y } \\
\text { procedimientos jurídicos }\end{array}$ & Doctor & $\begin{array}{l}\text { Facultad de Jurisprudencia } \\
\text { San Antonio de Abad }\end{array}$ \\
\hline \multicolumn{5}{|l|}{1943} \\
\hline $\begin{array}{l}\text { Pacheco } \\
\text { Garmendia, Elsa }\end{array}$ & $\begin{array}{l}\text { Aspectos del arte incaico. Música } \\
\text { y danza. }\end{array}$ & $\begin{array}{l}\text { Problema Indígena: Historia, } \\
\text { cultura y arte indígena/ } \\
\text { Organización y derecho } \\
\text { indígena }\end{array}$ & Doctor & $\begin{array}{l}\text { Facultad Letras, Sección } \\
\text { Historia San Antonio de Abad }\end{array}$ \\
\hline \multicolumn{5}{|l|}{1948} \\
\hline $\begin{array}{l}\text { Heredia Soto, } \\
\text { Florencio Daniel }\end{array}$ & $\begin{array}{l}\text { Informe arqueológico de kollkce - } \\
\text { wairachina, paucar cancha y atas } \\
\text { - kcasa. }\end{array}$ & $\begin{array}{l}\text { Problema Indígena: Historia, } \\
\text { cultura y arte indígena/ } \\
\text { Organización y derecho } \\
\text { indígena }\end{array}$ & Doctor & $\begin{array}{l}\text { Facultad Letras, Ciencias } \\
\text { Antropológicas San Antonio } \\
\text { de Abad }\end{array}$ \\
\hline \multicolumn{5}{|l|}{$S / F$} \\
\hline Cosío, Félix & $\begin{array}{l}\text { El curso de las civilizaciones } \\
\text { prehistóricas del Perú. }\end{array}$ & $\begin{array}{l}\text { Educación, diagnósticos, } \\
\text { enseñanza }\end{array}$ & Doctor & $\begin{array}{l}\text { Filosofía i Letras San Antonio } \\
\text { de Abad }\end{array}$ \\
\hline
\end{tabular}


La relación de tesis clasificadas hasta el momento, dan una gran cantidad de datos para diagnosticar la sociedad cusqueña con sus recursos intelectuales, sociales y naturales sobre todo para la segunda década del siglo XX.

Con el fin de motivar su consulta y dar un ejemplo de su utilidad, sugiero se lea mi tesis doctoral «Política educativa y ruralidad: 1900 a 1930». En este trabajo desarrollo el impacto de la pedagogía progresiva en la reforma educativa del Perú. Explico cómo la instrucción académica y profesional de los especialistas norteamericanos que llegaron a nuestro país, en especial la reforma emprendida por Alberto Giesecke en 1910 en el Cusco, contribuyó a la formación integral y humanista de los estudiantes para el conocimiento, investigación y desarrollo de su región. Una educación basada en los principios de la escuela activa, como la indagación, experimentación y conocimiento de su entorno, pero teniendo como punto de partida al individuo como sujeto transformador de su medio. Proceso que se inició ocho años antes que la reforma universitaria de Córdova y marcó un hito importante que fue parte de la demanda estudiantil de la reforma universitaria de Córdova, dicha demanda tuvo dos ejes centrales: la participación estudiantil en el gobierno de la universidad y la reforma pedagógica en la enseñanza universitaria. En ese sentido, en la primera mitad del siglo XX se evidencia una línea de continuidad entre la reforma universitaria del Cusco, la propuesta de la reforma pedagógica universitaria de José Antonio Encinas en 1931 y la reforma universitaria de 1946, donde participó Luis E. Valcárcel (ex alumno del Dr. Giesecke) como Ministro de Educación y José Antonio Encinas como senador en el gobierno de Luis Bustamante y Rivero (ex alumno del Dr. Giesecke).

Muchas de las tesis que pertenecen a esta colección me sirvieron como fuente para contextualizar las preocupaciones temáticas de los estudiantes del Cusco en la primera y segunda década del siglo XX. Tesis doctorales como la de E. Valcárcel en 1913 que trata sobre «La universidad del Cusco, su nueva organización», la de Bustamante y Rivero o José Luis Mejía que discuten en 1918 entorno a «La crisis universitaria»y «La organización de las escuelas rurales»,no hacen más que evidenciar que el proyecto educativo peruano y sus posteriores reformas, no solo fue una preocupación de la elite intelectual limeña, sino también, de la constancia y el esfuerzo de una generación de jóvenes cusqueños, liderado por un hombre como el Dr. Albert Giesecke, que apostó por la educación como la mejor herramienta de transformación social.

Cabe hacer una indicación final, referida a la ausencia de un tomo de las tesis empastadas por el Dr. Giesecke, presumo que ese tomo contiene las tesis etnográficas, lamentablemente ese tomo no fue hallado por mí. Los indicios que revelan la existencia de tesis etnográficas, se evidencian en que algunas están incluidas en la publicación digital de los primeros números de la Revista Universitaria. Queda pendiente ubicar las tesis mencionadas para completar la información que aquí se quiere consignar.

La conclusión más importante se refiere a reconocer que el valor de las tesis está en el significativo aporte que dichas tesis han hecho a la formación de profesionales de altísimo nivel, a partir de la creación de un conocimiento social, científico, crítico e integral y original sobre la realidad regional de Cusco de la segunda década del siglo XX, tanto en el campo de las humanidades como de las ciencias exactas. Todo ese esfuerzo se realizó con el objeto de promover el desarrollo regional cusqueño, considerando al conjunto de sus actores sociales: Indígenas, maestros, mujeres, empresarios, científicos, abogados; tal conocimiento fue complementado con un conjunto de propuestas insertadas para lograr los cambios deseados.

Ese conocimiento también fue la semilla de un gran movimiento social indigenista, que forjó la conciencia de compromiso social en toda una generación de intelectuales que participaron en 
los procesos políticos sociales de la época. Incluyendo al propio Doctor Giesecke quien llegó a ser Director General de Enseñanza entre 1924 y 1930, por el reconocimiento que le mereció su afecto, conocimiento, laboriosidad y compromiso con el país. Quien se basó en una pedagogía activa en la formación académica gestada en el diálogo y en la promoción humana integral de los estudiantes.

Anexos

ANEXO 1. CARÁTULA ACTUAL DE LA TESIS DOCTORAL DEL DOCTOR ALBERT A. GIESECKE EN UN LIBRO UBICADO EN LA BIBLIOTECA DE LA UNIVERSIDAD DE HARVARD.

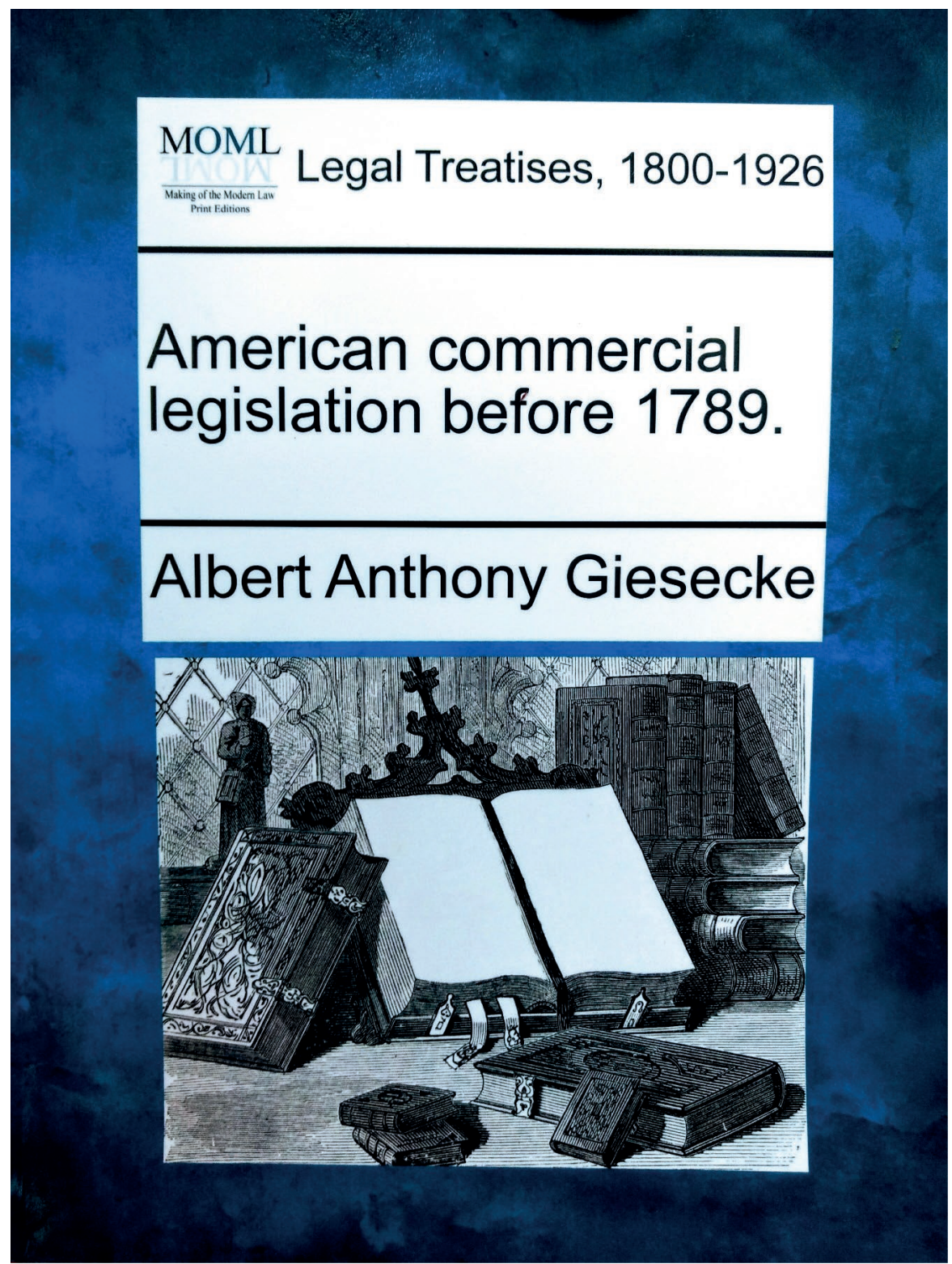


ANEXO 2. EJEMPLAR DE LA TESIS DE LUIS E. VALCÁRCEL «LA UNIVERSIDAD DEL CUSCO. SU NUEVA ORGANIZACIÓN» DE 1913 - BIBLIOTECA IRA - COLECCIÓN GIESECKE Carátula y página final con el $\mathrm{V}^{\circ} \mathrm{B}^{\circ}$ del Rector $\mathrm{Dr}$. A. Giesecke

\section{LUIS E. VALCARCEL}

\section{La Universidad del CuZco}

\section{SU NUEVA ORGANIZACION}

$$
-24-
$$

más elevados i de investigación a la universidad de Lima"? Apunta Mr. Bard las opiniones de Mrs. Brewn, de Nievil York, i Peterson, de Montreal: dice el $1^{\circ}$ : "Me inclino à pensar que una organización centralizada del sistema universitario sería deseable i utilizable en el Perá;" pero declara antes hon. radamente: "El conjunto de la organización de la educación depende de tal manera de las condiciones sociales i políticas del pueblo, que a su vez dependen cle la herencia de trarlicio. nes institucionales, que es imposible a la distancia hacer reco. mendaciones positivas respecto de los detalles de dichá orga. nización".

Si Mr. Brewn conociera tan bien nuestro país como lo conoce el rector de esta universidad, ex-catedrático de lá de Cornell, seguramente que retirairía su consejo. Está clemos. trado evidentemente que nuestra heterogeneidad étnica i territorial es insalvable; que no podemos lograr nada con la centralización i la uniformiclad a uitranza; que el ideal es la unificación mui diferente a las ilusiones de concentración.

Mr. Peterson es de parecer que "dentro del plan de una universidad federal las universidades menores podrian limitar. se a los dos primerus años de ciencias, letras i medicina i enviar a Lima a sus alumnos para continuar allí los estuclios i obtener sus grados"; pero Mr. Peterson ignora el sinnúme. ro de dificultades de todo orden que importa su idea. Ya no es nueva; ha sido combatida i derrotada hace tiempo.

Mr. Bard no puede negar que precisamente uno de los ob. jetos de la universiclad regional es la investigación científica, el estudio del medio ambiente tan rico en revelaciones pars la ciencia como el nuestro i si los grados superiores son los de especialización, es decir los de creación de ciencia, es saltante la necesidad de conservar i perfeccionar los grados superiores de la enseñanza de nuestra universidad.

Las preguntas 6 i 7 hállanse ya satistechas: sobre la fultima cabe decir que lis decanos i delegados de cada facultad serían suficientes para constituir el consejo universitario.

Cuzco, 19 de noviembre de 1912.

\section{Luis E. Valcârce!!}

$\mathrm{V}^{\circ} \mathrm{B}^{\circ}-$

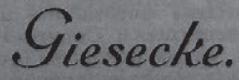

- 


\section{FUENTES DOCUMENTALES}

American Association of Anthropology. Certificado de membresía. George Grant Maccurdy. Secretary. YaleUniversityMuseum. New Haven, Comm. New Haven Comm, April 2 1912. (AG - 1275).

American Anthropological AssociationGeorge Grant Maccurdy. Secretary. YaleUniversityMuseum. New Haven, Conn.New Haven Comm, April $2^{\circ}$ 1912)(AG - 1275).

Board of Public Education. FirstSchool District of Pennsylvania. School of Commerce of the Central High School. Mayo 13, 1909. Permiso de licencia por dos años para Albert A. Giesecke.USA. (Fotocopia simple del original - Archivo personal)

Giesecke, A. (¿1951?). Albert Anthony Giesecke.[Documento mecanografiado]. Archivo del Instituto Riva-Agüero, Pontificia Universidad Católica del Perú, (Leg. AG-D-0158)

Jiménez, P. (27 de diciembre de 1923). Epístola dirigida al Sr. Dr. A. Giesecke. Colección Giesecke. Archivo del Instituto Riva-Agüero, Pontificia Universidad Católica del Perú, (AG - 0796).

\section{BIBLIOGRAFÍA}

Abbagnano, N. y Visalberghi, A. (1992). Historia de la pedagogía. España: Fondo de Cultura Económica.

Giesecke, M. (2015). Política educativa y ruralidad en el Perú: 1900 a 1930. Tomos I- II. Identificador digital (tesis doctoral). Lima: Universidad Nacional Mayor de San Marcos.

James, E. J. 1910 (1899). Commercial education. En Murray, N. (ed.). Education in the United States: A series of monographs. New Yprk: American Book Company

Kloppenberg, J. T. (1986). Uncertain Victory. Social Democracy and Progressivism in European and American Thought 1879 - 1920.New York: Oxford University Press.

Mason (Jr.), J. T. (1963). The Reminiscences of Albert A. Giesecke.Oral History Research Office (with Dr. Albert A. Giesecke in Lima, Perú). Universidad de Columbia, Mimeo.

\section{WEBGRAFÍA}

MINEDU. (2017). Nómina de Ministros. Recuperado en 26 de mayo de 2017dehttp://www.minedu.gob.pe/ institucional/nomina2.php

Velar, P. (2013). Albert A. Giesecke (1883-1968): La modernización indigenista de la Universidad. Recuperado en 9 de diciembre de 2017 de http://pavelvaler.blogspot.pe/2013/10/ albert-giesecke-18831968-la.html

Presentado: 21 junio 2017

Aceptado: 7 febrero 2018

Publicado online: 27 febrero 2018 